\title{
Une analyse engagée de la professionnalisation des pratiques d'évaluation
}

\author{
Benoît Gauthier \\ Réseau Circum inc., Réseau francophone de l'évaluation, École nationale \\ d'administration publique
}

\begin{abstract}
Résumé : Malgré un grand nombre de discussions autour de la notion de professionnalisation de la pratique de l'évaluation partout au monde, nombre de concepts associés ne sont pas clairement définis. Cette note fournit des définitions opérationnelles des notions de profession, professionnalisme, professionnel et professionnalisation et elle offre une méthodologie pour réfléchir aux tenants et aux aboutissants d'un processus national de professionnalisation. En conclusion, l'auteur analyse les perspectives qui souvrent pour la professionnalisation de la pratique de l'évaluation sur le plan international.
\end{abstract}

Mots-clés : définition, internationalisation, profession, professionnalisation

Abstract: Despite a great deal of discussion about the notion of professionalizing evaluation practice around the world, many associated concepts are not clearly defined. This practice note provides operational definitions of the concepts of profession, professionalism, professional and professionalization, and presents methodology designed to reflect on the ins and outs of a national process of professionalization. In conclusion, the author analyzes the prospects for the professionalization of the practice of evaluation at an international level.

Keywords: definition, internationalization, profession, professionalization

Cette note sur la recherche et la pratique se penche sur un sujet pris pour acquis en évaluation des programmes et des politiques, mais dont les conséquences logiques font l'objet de débats acrimonieux : la professionnalisation des pratiques d'évaluation. Peu nombreux sont ceux qui remettent en question l'aspect professionnel de la pratique de l'évaluation - qui admettrait ne pas être un "professionnel» ou une « professionnelle»-, mais plusieurs ne sont pas prêts à accepter les exigences de la professionnalisation. Comme nous le verrons, des conceptions artisanales et professionnalisées de l'évaluation s'affrontent. Nous annonçons que nous prenons fermement position en faveur de la professionnalisation de la pratique de l'évaluation après avoir analysé les processus nécessaires à ce cheminement et les risques et avantages associés.

Correspondance à l'auteur : Benoît Gauthier, 238 chemin Fleming, Cantley, Québec J8V 3B4; gauthier@circum.com 


\section{PROFESSION ET PROFESSIONNELS}

Profitez de la prochaine minute pour faire l'exercice suivant : demandez-vous si vous considérez être un professionnel et comment vous tirez cette conclusion. À partir de votre réponse, vous pourrez dessiner les contours de votre compréhension des notions de profession et de professionnel.

Depuis le Moyen Âge, le mot "profession » a été utilisé principalement pour les activités libérales - en particulier le clergé, les avocats et les médecins - par opposition au travail associé à la production ou au commerce. Les activités libérales nécessitaient une éducation avancée et une certaine forme de stage comme apprenti.

Certains disent que la grande période de la professionnalisation a eu lieu au $\mathrm{XIX}^{\mathrm{e}}$ siècle et au début du $\mathrm{XX}^{\mathrm{e}}$ siècle, lorsque les médecins et les avocats ont acquis un prestige et un pouvoir significatifs, et que d’autres groupes - ingénieurs, architectes, dentistes, enseignants, comptables, vérificateurs, infirmiers - ont commencé à se battre pour des positions similaires dans la société.

Au XX $X^{e}$ siècle, Larson (1977) a suggéré qu' « une profession est une occupation à temps plein qui apporte un statut élevé et un revenu confortable. Il repose sur une formation formelle dans un domaine de connaissances spécialisées, confirmée par quelque type de certification. Le professionnel fournit des services aux clients, pas des produits aux consommateurs, et gagne des honoraires plutôt qu'un salaire. Les membres d'une profession suivent un code de déontologie régi par des associations de professionnels plutôt que par l'État ou par un autre organisme extérieur ». Larson (1977) a suggéré que ces associations professionnelles tentent également de créer et de contrôler le marché des services de leurs membres, notamment en limitant la concurrence des praticiens non agréés.

Albisetti (2001) résume la position de Larson : « l’analyse de Larson contient des éléments d'interprétations bienveillantes et conspiratives de la professionnalisation qui existent dans toute la littérature savante. D’un côté, le processus apparaît comme la victoire de l'expertise, de l'honnêteté, voire du service désintéressé contre l'incompétence, la fraude et le charlatanisme. De l'autre, cela implique l'établissement d'un monopole, l'exclusion des non-professionnels et la limitation du choix du public. »

Tourmen (2007) distingue l'activité (l'exécution d'une série d'actions), la tâche (l'ensemble des buts et procédures prescrites, des performances exigées et des normes de qualité), le poste (le champ d'intervention sur le processus de travail, le positionnement d'interface avec les autres acteurs et la spécificité des démarches), le métier (formation spécifique, reconnaissance du métier par autrui et regroupement des personnes concernées) et la profession (forme historique d'organisation sociale, d'attribution d'identité et d'organisation du marché du travail et ensemble des savoir-faire spécifiques).

Dans le milieu de l'évaluation, les auteurs ne se sont pas appesantis sur la définition du concept de profession. Ils ont plutôt concentré leurs efforts sur les caractéristiques d'une profession établie. Par exemple, Worthen (1994) a proposé neuf de ces caractéristiques : 
1. Un besoin de spécialistes en évaluation.

2. Du contenu (connaissances et compétences) propre à l'évaluation.

3. Des programmes de préparation pour les évaluateurs.

4. Des occasions de carrière stables en évaluation.

5. Une certification ou un mécanisme de licence des évaluateurs.

6. Des associations professionnelles appropriées pour les évaluateurs.

7. L'exclusion de personnes non qualifiées de ces associations.

8. Une influence des associations d'évaluateurs sur les programmes de préparation destinés aux évaluateurs.

9. Des normes de pratique de l'évaluation.

Worthen (1994) a conclu que l'évaluation correspondait à six des neuf caractéristiques; absentes à l'époque étaient la certification, l'exclusion de personnes non qualifiées et l'influence sur les programmes de formation.

Dans le même esprit, mais beaucoup plus récemment, Picciotto (2011) a identifié les caractéristiques les plus fréquemment mentionnées d'une profession ${ }^{1}$ et énuméré les critères suivants pour évaluer le professionnalisme d’un groupe professionnel distinct:

1. Prestige et statut (demande élevée et croissante de services; récompenses financières substantielles; respectabilité et place reconnue dans les zones supérieures de léchelle professionnelle).

2. Dispositions éthiques (orientation en faveur de l'intérêt public, loyauté à l'égard du groupe professionnel; engagement à faire carrière tout au long de la vie, comportement respectueux, solidarité professionnelle; responsabilité à l'égard de la qualité du travail).

3. Expertise (éducation de haute qualité; exposition à la pratique, connaissances théoriques, compétences spécialisées, bon jugement, maîtrise des techniques).

4. Autonomie professionnelle (contrôle du recrutement, formation, directives professionnelles, normes éthiques, règles administratives, assurance de la qualité, procédures disciplinaires).

5. Titres de compétence (diplôme d'un établissement d'enseignement supérieur agréé; titre professionnel; résultats attestés; appartenance à des associations professionnelles).

Certains ont conclu des analyses d'auteurs comme Worthen et Picciotto que l'évaluation n'est pas une profession et, implicitement, qu'elle ne pouvait le devenir ou alors ne devrait pas le devenir. Pourtant, on peut lire ceci différemment : ces analyses tracent un programme permettant à l'évaluation de devenir une profession; elles nous indiquent ce que nous devons accomplir pour faire de l'évaluation une profession. Voilà qui mène à la notion de professionnalisation ou à ce qu'il faut faire pour se construire un statut professionnel. 


\section{PROFESSIONNALISATION}

En ce qui concerne l'émergence de professions (ou le processus de professionnalisation), Neal et Morgan (2000) suggèrent que le Royaume-Uni et l'Allemagne représentent deux approches opposées. Au Royaume-Uni, la professionnalisation s'est établie de bas en haut, des associations professionnelles au statut professionnel. En Allemagne, la professionnalisation s'est faite de haut en bas : une administration publique forte d'une jurisprudence donnant à l'État le contrôle de la définition des professions. Au Canada, il est évident que la professionnalisation s'est produite, en évaluation et ailleurs, selon le modèle britannique. Cependant, il est possible qu'une intervention étatique en faveur de la professionnalisation ait été concrétisée si les praticiens n'avaient pas pris la situation en main. Dans ce sens, le lancement du programme d'accréditation des évaluateurs par la Société canadienne d'évaluation (SCÉ) en 2010 représente une intervention depuis la base qui est profondément structurante (King, 2015; Dumaine, 2015).

Le professionnalisme est une convergence de connaissances, de valeurs, d'attitudes et de comportements. Un professionnel est une personne affichant les attributs du professionnalisme. La professionnalisation est une feuille de route, un voyage, un plan collectif visant à donner une importance particulière à une activité (ici, lévaluation) et à la reconnaître, afin de contribuer à une performance supérieure à cette activité et à la protection du public qui n'est pas en mesure de juger de la qualité de cette performance. Le professionnalisme est un état. La professionnalisation est un processus.

\section{LES COMPOSANTES DE LA PROFESSIONNALISATION}

Dans le monde de l'évaluation, les discussions sur la professionnalisation tendent à ne porter que sur un aspect : les mécanismes de la professionnalisation. Pourtant, on peut distinguer cinq classes d'ingrédients dans le parcours de professionnalisation : les conditions de l'écosystème, les mécanismes, le processus de changement, les ressources et le plan enraciné.

\section{Les conditions de l'écosystème d'évaluation}

La première classe d'ingrédients du parcours de la professionnalisation est l'écosystème national d'évaluation. Il s'agit de l'ensemble des circonstances qui définissent la situation actuelle et déterminent si un certain niveau de professionnalisation est conseillé et réalisable. Les ingrédients clés sont:

- le besoin pour l'expertise : existe-t-il une demande de travail de qualité dans le domaine visé?

- l'existence de l'expertise : existe-t-il un groupe identifiable d'individus qui pratiquent l'expertise?

- la perception d'un problème : existe-t-il un problème dans l'écosystème d'évaluation national qui se traduirait par un besoin de changement? 
- le niveau de consensus concernant le problème : existe-t-il un niveau relativement élevé d'accord sur la définition du problème, sa dynamique et les solutions possibles?

- les parties prenantes : existe-t-il des parties prenantes clés qui ont des intérêts clairs et forts en faveur ou contre la professionnalisation de lévaluation?

- l'environnement favorable : les structures institutionnelles, lois, politiques ou règlements, programmes universitaires de formation en évaluation, etc., favorisent-ils la professionnalisation?

\section{Les mécanismes de la professionnalisation}

La deuxième catégorie d'ingrédients du parcours de professionnalisation est celle dont on entend le plus parler : celle des mécanismes.

Parce que l'un des aspects essentiels du professionnalisme est la protection du public, les lignes directrices en matière déthique jouent un rôle important dans le processus de professionnalisation. L'élaboration de lignes directrices éthiques est généralement un marqueur précoce de la volonté de professionnaliser une pratique.

Le deuxième mécanisme le plus courant est lélaboration d'un ensemble de normes pour la pratique de lévaluation. Les normes parlent des caractéristiques d'un travail de qualité. Elles sont généralement énoncées au niveau des principes plutôt que dans les détails (Yarbrough, Shulha, Hopson et Caruthers, 2011).

Vient ensuite le développement de référentiels de compétences en tant que mécanisme de professionnalisation. Ces référentiels identifient les compétences requises pour une pratique professionnelle. Parmi ces compétences, certaines ne sont pas spécifiques à lévaluation : par exemple, un avocat doit être compétent en rédaction formelle et il en va de même pour un évaluateur. Plus d'une quinzaine de cadres de compétences pour la pratique de lévaluation ont été élaborés au cours des 10 ou 15 dernières années ${ }^{2}$. On y trouve des superpositions remarquables ainsi que des spécificités expliquées par les contextes.

Directives éthiques, normes de pratique et référentiels de compétences nont pas de sens s'ils ne sont pas promus, connus et utilisés. Ainsi, la communication et la formation constituent un autre mécanisme permettant de donner vie aux éléments de base. Plus généralement, le perfectionnement et la formation universitaire sont des mécanismes essentiels pour aider les évaluateurs à améliorer leurs compétences afin de découvrir de nouveaux outils, de nouveaux cadres, de nouvelles méthodes et de nouvelles approches. Les associations nationales dévaluation ainsi que les institutions d’enseignement ont un rôle important à jouer à cet égard.

Lorsque les quatre mécanismes précédents sont en place, le processus de professionnalisation est déjà en marche, mais il manque un élément clé : un moyen de reconnaître une conduite professionnelle. Selon la pratique contemporaine, cette reconnaissance peut provenir de différentes sources :

- Lachèvement d'une formation qui inclut lévaluation de l'apprentissage.

- La valorisation par les pairs comme dans le cas des programmes d'examen volontaire par les pairs de UKES et EES. 
- Laccréditation qui est fondé sur l'évaluation de la compétence attestée par la formation, l'expérience, le rendement, etc.

- La certification est une forme plus stricte de vérification de la compétence qui est généralement basée sur un examen.

- Les diplômes universitaires délivrés par les collèges et les universités.

- La délivrance d'une licence de travail encadrée par la loi.

Il y a des avantages et des inconvénients à chaque option, mais le message central est que la professionnalisation nécessite une forme de reconnaissance des praticiens individuels. Sans système de reconnaissance, le public ne dispose d'aucun moyen pour distinguer les praticiens dignes de confiance des fraudes potentielles.

Une fois un système de reconnaissance en place, il reste à créer un conseil de discipline : la logique veut que si la reconnaissance est étendue à un praticien, il devrait également être possible de l'enlever. La plupart des systèmes de reconnaissance basés sur des licences, voire tous ces systèmes, sont accompagnés d'un certain type de conseil de discipline.

\section{Le processus de changement}

La troisième classe d'ingrédients du parcours de professionnalisation est le processus de changement. La professionnalisation signifie le changement - de l'informel au structuré, de désorganisé à organisé, d'indéfini à explicite - et peut donc être intégrée à un cadre de gestion du changement. Heureusement, il existe de nombreux ouvrages et documents sur la gestion du changement et ils tendent à s'entendre sur les étapes à prendre en compte : l'analyse préliminaire et contextuelle; l'identification des facilitateurs et des obstacles; la planification des actions; le suivi; et les ajustements nécessaires de cadence, rythme et contenu.

La stratégie de changement doit être à la mesure de la capacité à la mettre en œuvre. Élaborer un plan ouvert, transparent, participatif, inclusif et ambitieux (mais réaliste) constitue également un moyen de se rapprocher des partenaires dans la stratégie de changement et de professionnalisation.

\section{Les ressources}

La quatrième classe d'ingrédients du parcours de professionnalisation concerne les ressources - au plus simple, l'argent et le temps - qui sont nécessaires pour s'engager dans la voie de la professionnalisation. Bien entendu, les ressources requises dépendent de l'exhaustivité du parcours de professionnalisation.

\section{Un plan enraciné}

Cette analyse du contexte national et une gestion prudente du changement devraient aboutir à une solution ou à un plan de professionnalisation enraciné, adapté au contexte national, reflétant les forces en jeu et proportionné aux ressources disponibles. Dans l'idéal, ce plan enraciné devrait aboutir au déploiement d'un ensemble complet des caractéristiques professionnelles identifiées plus tôt. 


\section{BÉNÉFICES, RISQUES ET ARBITRAGES ASSOCIÉS À LA PROFESSIONNALISATION}

Jacob et Boisvert (2010), Picciotto (2011), Evetts (2018) et d'autres ont fourni des informations utiles sur les bénéfices et les risques de la professionnalisation.

\section{Bénéfices}

- La construction d'une identité partagée à travers des définitions communes, une terminologie, des références, des modèles, des théories, etc.

- La promotion d'une image positive auprès des clients et des consommateurs préférant généralement traiter avec un professionnel plutôt qu'avec un amateur.

- L'amélioration de la formation en évaluation et des connaissances chez les évaluateurs.

- Une formation avancée influencée par la pratique et par les compétences afin que les professionnels émergents puissent plus facilement trouver une place dans la profession.

- Une pratique d'évaluation plus homogène, interprétée comme un avantage en ce qu'elle permet de mieux comprendre ce que font les évaluateurs.

- Une qualité d'évaluation améliorée à la suite d'une prise de conscience accrue et d'une formation améliorée.

- Une utilisation accrue des données probantes issues de l'évaluation grâce à des processus mieux gérés et à des études plus pertinentes.

- Plus de prestige pour les évaluateurs qui peuvent se référer à un ensemble de compétences pour présenter leur activité.

- Une réduction de l'incidence des comportements problématiques grâce à une meilleure formation et au respect des normes et des directives.

- La protection du public découlant d'une meilleure définition de l'activité des évaluateurs et de l'amélioration des capacités professionnelles.

\section{Risques}

- La glorification des opinions de quelques-uns au lieu d'une perspective consensuelle si le processus de professionnalisation est mal géré et que les positions d'un petit groupe dominent la conversation.

- Une diversité méthodologique restreinte si les normes ne permettent pas l'innovation.

- Un champ restreint si l'innovation n'est pas correctement valorisée.

- La rigidification de la formation normalisée si les normes sont trop strictes.

- L'exclusion de praticiens talentueux si le processus de professionnalisation crée des barrières excessives à l'entrée.

- Faire passer les intérêts des évaluateurs avant ceux des clients si le corporatisme prime sur la recherche de la qualité. 
Avec certains auteurs déjà cités, je pose que ces risques sont gérables grâce à un processus de discussion ouvert, transparent et démocratique.

\section{Arbitrages}

Dans le cadre d'une discussion sur la nécessité de professionnaliser les spécialistes en cybersécurité, le National Research Council (2013) a exposé certains arbitrages qu'ils ont associés à la professionnalisation et qui pourraient être pertinents pour notre discussion.

1. Qualité contre quantité. Il est possible que l'amélioration de la qualité se fasse au prix de restrictions d'accès créées par des barrières à l'entrée. Ceci est plus probable si le parcours de professionnalisation comprend des étapes obligatoires comme l'exigence d'une formation ou d'une certification. L'expérience de la SCÉ avec son programme d'accréditation a plutôt consisté à attirer les nouveaux praticiens qui pouvaient désormais suivre un chemin professionnel menant à l'évaluation.

2. Normalisation contre dynamisme. Les certifications ou cursus communs fournissent un certain niveau de normalisation qui aide à définir le domaine. Le compromis pourrait être une réduction du dynamisme ou de l'innovation dans un contexte de changement rapide. Cet effet négatif n’a pas été observé dans le contexte canadien. Au contraire, le cadre de compétences de la SCÉ a été révisé 10 ans après sa configuration initiale; il inclut maintenant des considérations sur l'inclusion, le partage du pouvoir et les droits de la personne, lesquelles nétaient pas incluses initialement.

3. Meilleure information des employeurs contre fausse certitude. Les mécanismes de reconnaissance officiels offrent une certaine capacité à évaluer les individus, mais une confiance excessive en ceux-ci peut exclure certaines des personnes parmi les plus talentueuses et les plus aptes. Ce risque n'est pas différent de celui d'une utilisation aveugle des diplômes universitaires.

4. Capacités du travailleur contre exigences du poste. Les capacités génériques liées aux compétences ne correspondent pas nécessairement aux exigences spécifiques du poste. Par exemple, un évaluateur spécialisé dans l'analyse qualitative et accrédité peut ne pas répondre aux exigences d'une évaluation de résultats quantitative sans compromis. Cependant, les mécanismes de professionnalisation n'existent pas pour assurer une telle correspondance.

5. Stimulation contre restriction de l'offre. La professionnalisation peut augmenter l'offre au fil du temps, car elle contribue à accroître la notoriété et le caractère souhaitable d'une profession, mais elle peut également réduire le nombre de candidats futurs si les exigences sont dépassées ou excessives. 


\section{VERS L'INTERNATIONALISATION DE LA PROFESSION D'ÉVALUATION}

Jusqu'ici, l'analyse a porté sur un contexte national. Il est en effet plus facile d'analyser, de planifier et d'agir dans un ensemble plus homogène que de le faire dans un environnement très hétérogène. Ainsi, un niveau d'intervention national semble approprié. Au moins au début. Mais est-il concevable qu'il y ait une voie vers la professionnalisation à l'échelle mondiale? D’autres l'ont fait. Par exemple, le titre de conseiller en management certifié (CMC) est reconnu partout même s'il est géré localement ou nationalement sur la base de critères et de processus approuvés internationalement.

IDEAS, l'association internationale d'évaluation du développement, une association mondiale d'évaluateurs individuels, a élaboré un " code d'éthique » et des « compétences pour les évaluateurs, les gestionnaires et les commissaires en évaluation du développement ". Ce sont des documents (mécanismes) de portée internationale. En un sens, le cadre de compétences du Groupe d'évaluation des Nations-Unies (GENU, 2016) a également une portée mondiale. De plus, il est remarquable que tous les codes d’éthique relatifs à l'évaluation partagent de nombreuses considérations. Les normes adoptées par certaines sociétés d'évaluation s'inspirent des travaux du Joint Committee on Standards for Educational Evaluation (JCSEE). Et les cadres de compétences existants se sont considérablement influencés mutuellement. Une comparaison récente de quatre référentiels de compétences publiés depuis 2016 démontre que, au niveau micro de chaque compétence, environ $50 \%$ des compétences étaient identiques dans quatre ou cinq de ces cadres.

Tracer une voie mondiale de professionnalisation ne serait pas un mince exploit, mais, même si le résultat était imparfait par manque d'une base solide et d'un contexte simple, cela constituerait une source d'inspiration importante pour les efforts nationaux.

\section{NOTES}

1 Possession d'une compétence basée sur des connaissances théoriques; offre de formation et d'éducation; vérification de la compétence des membres; organisation professionnelle; respect d'un code de conduite; et service altruiste.

2 American Evaluation Association, https://www.eval.org/page/competencies; Aotearoa New Zealand Evaluation Association, http://www.anzea.org.nz/wp-content/ uploads/2013/05/110801_anzea_evaluator_competencies_final.pdf; Australian Evaluation Society, https://www.aes.asn.au/resources/evaluator-competencies.html; Canadian Evaluation Society, https://evaluationcanada.ca/competencies-canadian-evaluators; CLEAR-AA; Department of Performance Monitoring and Evaluation, South Africa, http://old.samea.org.za/index.php? module=MediaAttach\&func=download\&fileid=288; European Evaluation Society, https://www.europeanevaluation.org/sites/ default/files/surveys/EES\%20EVALUATION\%20CAPABILITIES\%20FRAMEWORK. pdf; German Evaluation Association, https://www.degeval.org/en/publikationen/ requirement-profiles-for-evaluators/; International Development Evaluation Association, 
https://ideas-global.org/the-competencies-framework/; International Finance Corporation; Japanese Evaluation Society; Latin American Region Coordinating Team, To "Leave No One Behind": Towards Capacity Building in Gender Transformative Evaluation with Cultural Relevance in Latin America; Swiss Evaluation Society, https://www. seval.ch/fr/standards-competences/competences/; Treasury Board Secretariat of Canada, https://www.canada.ca/en/treasury-board-secretariat/services/audit-evaluation/ centre-excellence-evaluation/leadership-competencies-federal-heads-evaluation.html; United Kingdom DFID Department for International Development; Western Michigan University Interdisciplinary Ph.D. in Evaluation Program, https://wmich.edu/ evaluationphd/about; World Bank Independent Evaluation Group (IEG), http://ieg. worldbankgroup.org/topic/evaluation-capacity-development

\section{BIBLIOGRAPHIE}

Albisetti, J. C. (2001). Professionals and professionalization. Consulté au https://www. encyclopedia.com/international/encyclopedias-almanacs-transcripts-and-maps/professionals-and-professionalization

Dumaine, F. (2015). A point of no return finally reached: The journey ahead. La Revue canadienne dévaluation de programme, 29(3), 154-159. https://doi.org/10.3138/ cjpe.29.3.154

Evetts, J. (2018). Professions in turbulent times: Changes, challenges and opportunities. Sociologia, Problemas e Práticas, 88, 43-59. https://doi.org/10.7458/SPP20188814797

Groupe d'évaluation des Nations-Unies (GENU). (2016). Professionalization of evaluation: Concept paper. Consulté au http://www.uneval.org/document/download/2611

Jacob, S. et Boisvert, Y. (2010). To be or not to be a profession: Pros, cons and challenges for evaluation. Evaluation, 16(4), 349-369. https://doi.org/10.1177/1356389010380001

King, J. A. (2015). From the outside, looking in with a smile: A summary and discussion of CES's credentialed evaluator designation. La Revue canadienne d'évaluation de programme, 29(3), 134-153. https://doi.org/10.3138/cjpe.29.3.134

Larson, M. S. (1977). The rise of professionalism: A sociological analysis. Berkeley, CA: University of California Press.

National Research Council. (2013). Professionalizing the nation's cybersecurity workforce?: Criteria for decision-making. Washington, DC: The National Academies Press. Consulté au https://www.nap.edu/catalog/18446/professionalizing-the-nations-cybersecurityworkforce-criteria-for-decision-making

Neal, M. et Morgan, J. (2000). The professionalization of everyone? A comparative study of the development of the professions in the United Kingdom and Germany. European Sociological Review, 16(1), 9-26. https://doi.org/10.1093/esr/16.1.9

Picciotto, R. (2011). The logic of evaluation professionalism. Evaluation, 17(2), 165-180. https://doi.org/10.1177/1356389011403362

Tourmen, C. (2007). Activité, tâche, poste, métier, profession : quelques pistes de clarification et de réflexion. Santé Publique. 19, 15-20.

Worthen, B. R. (1994). Is evaluation a mature profession that warrants the preparation of evaluation professionals? New Directions for Program Evaluation, 62, 3-15. https:// doi.org/10.1002/ev.1672 
Yarbrough, D. B., Shulha L. M., Hopson, R. K. et Caruthers, F. A. (2010). The program evaluation standards: A guide for evaluators and evaluation users (3rd edition). Thousand Oaks, CA: Corwin Press. Consulté au https://jcsee.org/program/

\section{PRÉSENTATION D'AUTEUR}

Benoît Gauthier est évaluateur qualifié. Il a été vice-président et président de la SCÉ, vice-président et président du RFE et vice-président de l'OICE ainsi que membre du comité de gestion d'EvalPartners. Il détient des diplômes avancés en science politique et en administration publique de l'Université Laval et de l'École nationale d'administration publique du Québec. Il agit comme consultant en évaluation et comme formateur universitaire en recherche sociale. Il est co-éditeur du manuel intitulé Recherche sociale, de la problématique à la collecte des données et auteur de plusieurs articles. 